\title{
Amplification of the Capacitance Containing Nematic Liquid Crystal Embedded with Metal Nanoparticles
}

\author{
Shunsuke Kobayashi, ${ }^{1}$ Yoshio Sakai, ${ }^{2}$ Tomohiro Miyama, ${ }^{1}$ \\ Naoto Nishida, ${ }^{3}$ and Naoki Toshima ${ }^{3}$ \\ ${ }^{1}$ Liquid Crystal Institute and Department of Electrical Engineering, Tokyo University of Science, Yamaguchi, Japan \\ ${ }^{2}$ Department of Engineering and Science, Tokyo University of Science, Yamaguchi, Japan \\ ${ }^{3}$ Advanced Material Institute and Department of Applied Chemistry, Tokyo University of Science, Yamaguchi 1-1-1, Daigaku-dori, \\ Sanyo-Onoda, Yamaguchi 756-0884, Japan \\ Correspondence should be addressed to Shunsuke Kobayashi, kobayashi@rs.tus.ac.jp
}

Received 11 August 2011; Revised 9 November 2011; Accepted 11 November 2011

Academic Editor: Zhi Li Xiao

Copyright () 2012 Shunsuke Kobayashi et al. This is an open access article distributed under the Creative Commons Attribution License, which permits unrestricted use, distribution, and reproduction in any medium, provided the original work is properly cited.

\begin{abstract}
Herein, we report the dielectric properties of liquid crystal cells embedded with the nanoparticles of Pd, where each of which is covered with a diffusion cloud. It is shown that an amplification of the capacitors with these media occurs with the gain, $A_{c}=12.5$, when the concentration of nanoparticles is $0.3 \mathrm{wt} \%$ and in the frequency region below the dielectric relaxation frequency, $158.5 \mathrm{~Hz}$. This phenomenon is explained by an equivalent circuit model together with a compatible explanation of the dielectric strength and the relaxation time. It is claimed that the occurrence of the capacitance amplification may be attributed to a special nature of the oscillating extra charges, which appear in the region between the host medium and inclusion, and produces an effective negative dielectric constant of the special nanoparticles. This explanation was made by formulating an independent auxiliary equivalent circuit equation that enables to determine the numerical condition of the production of the negativity in the dielectric constant of inclusions (nanoparticles), and, thus, we succeeded in getting the numerical value of this dielectric constant and that of the gain of the capacitance amplification.
\end{abstract}

\section{Introduction}

We synthesized the nanoparticles of metal such as palladium, $\mathrm{Pd}$, and $\mathrm{Ag}$ that are covered with the surrounding nematic liquid crystal (NLC) molecules, and these nanoparticles are embedded in an NLC medium having twisted nematic structure, where the adopted synthesizing methods were those of alcohol reduction or UV irradiation [1].

Depending on the difference of synthesizing method and the properties of host NLCs, we had two kinds of NLCs embedded with the nanoparticles of Pd. In the type (1), the medium exhibits the Debye type dielectric function with a dielectric relaxation frequency, $f_{R}$, that ranges from $100 \mathrm{~Hz}$ to $10 \mathrm{kHz}$ depending on the nanoparticle concentration, and it was shown that liquid crystal electrooptical (EO) devices with TN structure fabricated using the type (1) NLC exhibits a frequency modulation EO response with a short response times [2-4]. This phenomenon is thought to be attributed to increase of the dielectric strength that corresponds to the amplification of the dielectric constant of the nanoparticleembedded NLC medium at $f<f_{R}$. On the other hand, NLC media of the type (2) exhibit a low dielectric relaxation frequency, say, at $f_{R} \approx 10 \mathrm{~Hz}$, and no amplification in the dielectric constant occurs. An LC-EO device such as an STNLCD using the NLC medium of the type (2) exhibits a fast response speed by three times compared to those without doping nanoparticles at a low temperature, say, at $-20^{\circ} \mathrm{C}[5]$. The essential difference between the type (1) and the type (2) is that, in the former, each nanoparticle is covered with an ionic diffusion cloud, contrary to this in the type (2), nanoparticles are bare and just surrounded by NLC molecules and not covered with a diffusion cloud.

In a previous paper, we discussed the concentration dependence of the dielectric relaxation time in an NLC 
corresponding in the type (1) and we obtained a good agreement between the experimental data and theoretical calculation based on an equivalent circuit model [6]. Herein, the present paper discusses the compatibility between the dielectric relaxation time and the dielectric strength using an extended equivalent circuit model, the reason for establishing this model is that conventional theories on the heterogeneous dielectric medium such as by Maxwell-Garnett $[7,8]$ and Maxwell [9] and Wagner [10] (M-W theory) are unable to explain the experimentally obtained relaxation times that depend on the concentration of nanoparticles.

In this paper, the increase of the dielectric strength from a base line in the Debye function, which is caused by doping nanoparticles, is taken as an amplification of the dielectric constant and hence that of the capacitance of NLC cell.

A capacitor, which is dealt with in this paper, comprises not only an inhomogeneous liquid crystal host medium but also the layered structures such as liquid crystal alignment layers.

Oka introduced an equivalent circuit theory that enables to deal with multilayered inhomogeneous capacitors [11]. The theoretical analysis adopted in this paper is an extension of Oka's approach to a general system comprising both the heterogeneous host medium and layered substrate interfacial structures.

\section{Experimental Methods}

We fabricated twisted nematic (TN) liquid crystal cells using nematic liquid crystal (NLC), 4-4' pentylcyanobiphenyl, $5 \mathrm{CB}$, as a host medium that is embedded with the nanoparticles of metal, Pd [1]. The size of the nanoparticles is $3 \mathrm{~nm}$ with the standard deviation of $0.4 \mathrm{~nm}$.

The dimension of TN cells is as follows: the size of transparent electrodes is $8 \mathrm{~cm} \times 8 \mathrm{~cm}$, and the cell gap is $5 \mu \mathrm{m}$. NLCs, $5 \mathrm{CB}$ without doping chiral molecules, are aligned on the surface alignment polyimide layers (SE-130, Nissan Chem. Ind.) by rubbing. We measured the frequency dependence of capacitances of the sample cells with a measuring instrument, an LCR meter, (Hioki, Model 352250 ) at $25^{\circ} \mathrm{C}$, where the relative error in the measurement of dielectric constant was $0.1 \%$.

The measurement of the dielectric constant of TN cells was done with an AC voltage of with the amplitude of $100 \mathrm{mV}$, which is below the threshold voltage, $V_{\text {th }}=1.8 \mathrm{~V}$, where the measurements were done without applying bias voltage. Thus, the measurement was done on TN cells under their threshold voltage. This means that the NLC media have a planar configuration.

\section{Experimental Results}

Figures 1 and 2 show the real part, $\varepsilon^{\prime}(\omega)$, and the imaginary part of dielectric constant, $\varepsilon^{\prime \prime}(\omega)$, of TN-LCD cells doped with nanoparticles (samples A through D that are indicated on Table 1), where the solid lines are drawn as the calculated curves obtained by performing the Cole-Cole plot [12] that produces an effective plot of $\varepsilon^{\prime}$ versus $\varepsilon^{\prime \prime}$ as shown in Figure 3, where the frequency is a parameter. This plot is

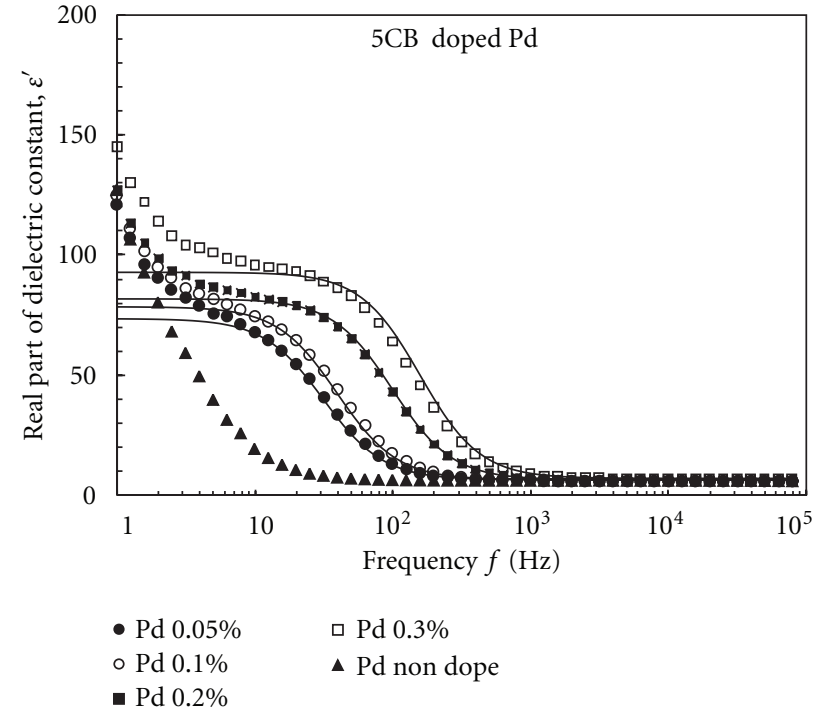

FIGURE 1: Frequency dependence of the real part of dielectric constants, where the solid lines are drawn by performing the ColeCole plots.

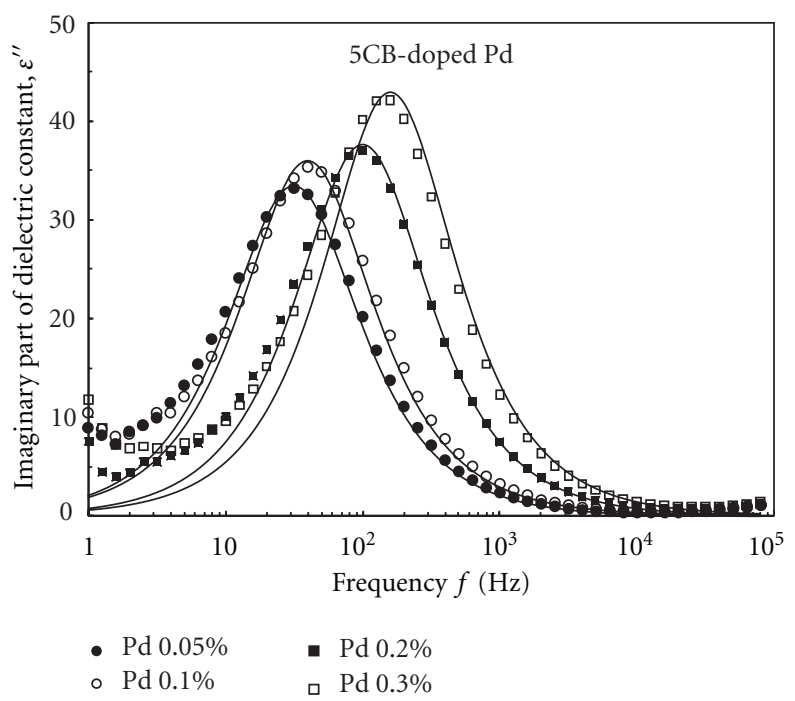

FIGURE 2: Frequency dependence of the imaginary part of dielectric constants, where the solid lines are drawn by performing the ColeCole plots.

useful to avoid the effects of mobile ions occurring at very low frequency region below $10 \mathrm{~Hz}$ as is seen in Figure 1 . Furthermore, this plot makes it possible to evaluate the true value of $\varepsilon(0)$ as shown by the solid lines in Figure 1. The relevant dielectric data on these sample cells, (A) through (D), are shown in Table 1 . The data of $\varepsilon^{\prime}(\omega)$ of a cell without doping nanoparticle is not shown in the Table 1, but this is indicated by triangles in Figure 1: the $\varepsilon^{\prime}(\omega)$ of this kind of cell always lies on a horizontal with $\varepsilon=6.5$ over all the frequency range from the several tens $\mathrm{Hz}$ to the several ten thousands $\mathrm{Hz}$ expect below about $20 \mathrm{~Hz}$. This value is equivalent to $\varepsilon(\infty)$. According to an independent measurement, similar 
TABLE 1: Dielectric properties of nematic liquid crystal doped with the nanoparticles of Pd.

\begin{tabular}{lccccccccc}
\hline Samples & $\begin{array}{c}\text { Concentration } \\
(\text { wt } \%)\end{array}$ & $\begin{array}{c}\text { Volume } \\
\text { occupation factor } \\
\phi_{2}\end{array}$ & $\begin{array}{c}\text { Dielectric relaxation } \\
\text { frequenc } f_{R}(\mathrm{~Hz})\end{array}$ & $\begin{array}{c}\text { Dielectric } \\
\text { relaxation time } \\
\tau(\mathrm{ms})\end{array}$ & $\varepsilon\left(f_{R}\right)^{*}$ & $\varepsilon(0)$ & $\varepsilon(\infty)$ & $\begin{array}{c}\text { Jump } \\
\varepsilon(0)-\varepsilon(\infty)\end{array}$ & $\begin{array}{c}\text { Gain } \\
\{\varepsilon(0)-\varepsilon(\infty)\} / \varepsilon(\infty)\end{array}$ \\
\hline $\mathrm{A}$ & 0.05 & $4.58 \times 10^{-5}$ & 31.6 & 5.04 & 39.9 & 73.4 & 6.4 & 67.0 & 10.5 \\
$\mathrm{~B}$ & 0.1 & $9.15 \times 10^{-5}$ & 39.8 & 4.00 & 42.3 & 78.3 & 6.3 & 72.0 & 11.4 \\
$\mathrm{C}$ & 0.2 & $1.83 \times 10^{-4}$ & 100.0 & 1.59 & 44.3 & 82.0 & 6.6 & 75.4 & 11.5 \\
$\mathrm{D}$ & 0.3 & $2.75 \times 10^{-4}$ & 158.5 & 1.00 & 49.9 & 92.9 & 6.9 & 86.0 & 12.5 \\
\hline
\end{tabular}

* The values of $\varepsilon\left(f_{R}\right)$ are calculated with $\varepsilon\left(f_{R}\right)=\{\varepsilon(0)+\varepsilon(\infty)\} / 2$.

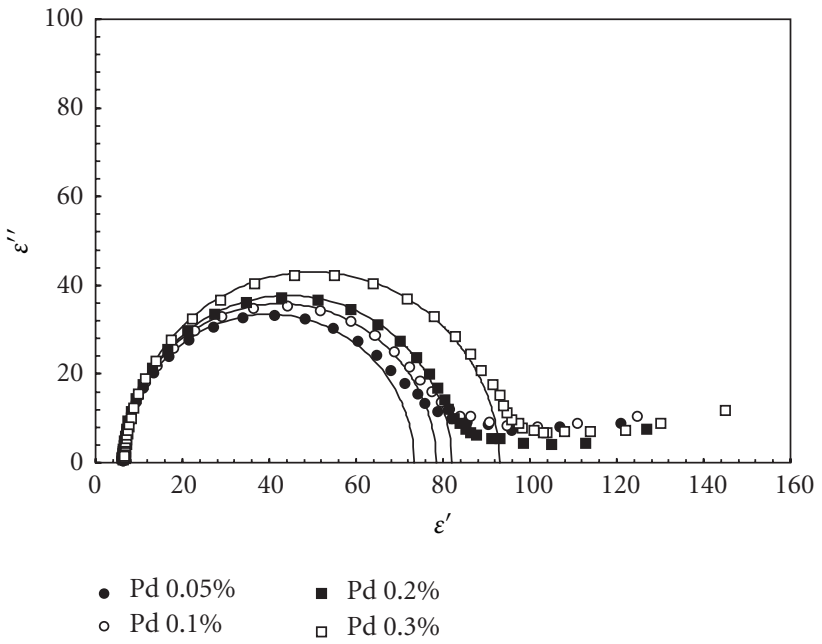

Figure 3: The Cole-Cole plots on our nanoparticle-embedded NLC, 5CB system.

results are obtained on samples with $5 \mathrm{CB}-\mathrm{Ag} / \mathrm{Pd}$ and $5 \mathrm{CB}-$ Ag nanoparticles.

\section{Analytical Consideration Based on an Equivalent Circuit Model}

Historically, the dielectric dispersion of a heterogeneous dielectric medium has been analyzed using the theory by Maxwell-Garnett [7, 8], Maxwell [9], and Wagner [10]. Despite the dependence on the concentration of nanoparticles of dielectric function, these theories are lacking in the compatibility between the dielectric strength, $\varepsilon(0)-\varepsilon(\infty)$, and the dielectric relaxation time, $\tau$, that are presented in Table 1. This discrepancy between the traditional theories and ours may be due to the difference of the nature of objects. Our objective is liquid-crystal cells that comprise not only the heterogeneous host media but also the layered structures in the substrates such as liquid crystal surface alignment layers. To resolve this problem, we explored several approaches and after that we came up with an idea to formulate an equivalent circuit model by extending Oka's treatment as shown in Figures 4(a) and 4(b) and to take into account the surface interfacial effect. In a previous paper, we formulated an equivalent circuit theory of heterogeneous dielectric medium and investigated the relaxation time [5]. The whole behavior of the relaxation time, which decreases with the increasing of the nanoparticle concentration, is not largely influenced by the surface effect as will be discussed in the Section 4.2. In this paper, we discuss compatibility between the dielectric strength, which is called gain, and the relaxation time for the actual concentration of nanoparticles.

4.1. The Case Where Only the Bulk Effect Is Considered. First, we shall conduct an analytical consideration on the bulk NLC medium with nanoparticles without taking account of alignment layers existing in an LC cell; later on, we consider the interfacial effect of the alignment layers on the dielectric function in our dielectric system.

First, we suggest a model by assuming that the nanoparticles locate regularly with an average distance as shown in Figure 4(a) even though their actual distribution may be at random. The mean distance between nanoparticles is evaluated through the concentration of nanoparticles as follows: the weight percent of nanoparticles is

$$
[\mathrm{wt} \%]=\frac{N v}{V} \cdot \frac{\rho_{M}}{\rho_{\mathrm{LC}}},
$$

where $N v / V$ is the volume occupation factor $\phi_{2}$, and $\rho_{M}$ and $\rho_{\text {LC }}$ are the specific gravity of metal nanoparticle and that of NLC medium, respectively. Then, $N=[\mathrm{wt} \%]\left(\rho_{\mathrm{LC}} / \rho_{M}\right)(V / v)$ and $\ell^{3} N=V$, where $\ell$ is the mean distance between nanoparticles, and then $\ell=(V / N)^{1 / 3}$. In our system, $\ell$ is about $50 \mathrm{~nm}$. We take a cube called a subunit having the volume of $\ell^{3}$ that contains an individual nanoparticle (Figure 4(a)); we set an equivalent circuit for this unit as shown in Figure 4(b), where $G_{1}$ and $C_{1}$ are the conductance and the capacitance of the liquid medium; $G_{2}$ and $C_{2}$ are those of an inclusion that is actually covered with an ionic diffusion cloud.

The conductance of each part is then written as $G_{1}=$ $\sigma_{1}\left(\ell^{2} / \ell\right)$ and $G_{2}=\sigma_{2}\left(a^{2} / a\right)$, where we assume that the nanoparticle has a cubic shape with the volume of $a^{3}$. If we assume that metal nanoparticle has a spherical form, then we meet a difficulty in defining the capacitance and conductance of this spherical metal particle.

From the circuit shown in Figure 4(b), the admittance of this subsystem is

$$
Y=\frac{\left(G_{1}+i \omega C_{1}\right)\left(G_{2}+i \omega C_{2}\right)}{\left(G_{1}+G_{2}\right)+i \omega\left(C_{1}+C_{2}\right)}
$$




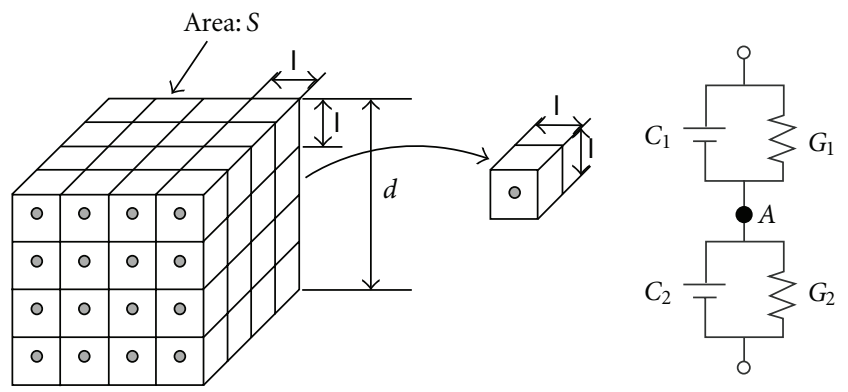

(a) Crystal mode

(b) Sub-unit and an equivalent circuit

FIgURE 4: Crystal model and equivalent circuit model of our system.

The imaginary part of (2) is $\omega C$, and then the capacitance of the system, $C$, reads

$$
C=\frac{\left(G_{1}+G_{2}\right)\left(C_{1} G_{2}+C_{2} G_{1}\right)-\left(G_{1} G_{2}-\omega^{2} C_{1} C_{2}\right)\left(C_{1}+C_{2}\right)}{\left(G_{1}+G_{2}\right)^{2}+\omega^{2}\left(C_{1}+C_{2}\right)^{2}} .
$$

This has a frequency dispersion called that of the Debye type:

$$
C(\omega)=C(\infty)+\frac{C(0)-C(\infty)}{1+\omega^{2} \tau^{2}}
$$

with

$$
\begin{aligned}
\tau & =\frac{C_{1}+C_{2}}{G_{1}+G_{2}}, \\
C(\infty) & =\frac{C_{1} C_{2}}{C_{1}+C_{2}} \\
& =C_{1} .
\end{aligned}
$$

When no nanoparticles exist, $C(0)$ will be

$$
C(0)=\frac{\left(G_{1}+G_{2}\right)\left(C_{1} G_{2}+C_{2} G_{1}\right)-G_{1} G_{2}\left(C_{1}+C_{2}\right)}{\left(G_{1}+G_{2}\right)^{2}},
$$

thus, we have

$$
C(0)-C(\infty)=\frac{\left(C_{1} G_{2}-C_{2} G_{1}\right)^{2}}{\left(G_{1}+G_{2}\right)^{2}\left(C_{1}+C_{2}\right)} .
$$

The numerator of (8) is originated from the difference of the RC time constants such that $\Delta \tau=\tau_{1}-\tau_{2}=R_{1} C_{1}-R_{2} C_{2}=$ $C_{1} / G_{1}-C_{2} / G_{2}=\left(C_{1} G_{2}-C_{2} G_{1}\right) / G_{1} G_{2}$. This is the origin of the jump $C(0)-C(\infty)$ that is the dielectric strength and also called the Maxwell-Wagner (M-W) effect.

Now, let us evaluate the capacitance of the whole system by making the parallel and series summation of the subunits illustrated in Figures 3(a) and 3(b).

The number of cubes with the volume of $\ell^{3}$ is $L / \ell$ along the vertical direction of an LCD cell and there are $S / \ell^{2}$ cubes on the surface of the cell, where $S$ and $L$ are the area and thickness of the LC layer. Thus, by making the summation of series and parallel circuit composition, we have a shape factor $S / L \ell$. Further, using dielectric constant $\varepsilon_{i}$ and the conductivity $\sigma_{i}$ of each element, we have $C_{1}=\varepsilon_{1}\left(\ell^{2} / \ell\right), G_{1}=$ $\sigma_{1}\left(\ell^{2} / \ell\right)$ for liquid crystal and $C_{2}=\varepsilon_{2}\left(a^{2} / a\right), G_{2}=\sigma_{2}\left(a^{2} / a\right)$ for a nanoparticle (inclusion).

Then, the total capacitance, $C$, is given as $C=\varepsilon(S / L)$, where $\varepsilon$ is the mean value of the dielectric constant of the whole system.

Thus, from (4), the Debye dispersion formula is derived as follows:

$$
\varepsilon(\omega)=\varepsilon(\infty)+\frac{\varepsilon(0)-\varepsilon(\infty)}{1+\omega^{2} \tau^{2}} .
$$

Equations (5) and (8) give a formulae for the relaxation time:

$$
\begin{aligned}
\tau & =\frac{\varepsilon_{0}\left(\varepsilon_{1}+\varepsilon_{2} \phi_{2}^{1 / 3}\right)}{\sigma_{1}+\sigma_{2} \phi_{2}^{1 / 3}} \\
& =\tau_{1} \frac{1+\left(\varepsilon_{2} / \varepsilon_{1}\right) \phi_{2}^{1 / 3}}{1+\left(\sigma_{2} / \sigma_{1}\right) \phi_{2}^{1 / 3}} \\
& =\tau_{1} \frac{1-\left|\varepsilon_{2} / \varepsilon_{1}\right| \phi_{2}^{1 / 3}}{1+\left(\sigma_{2} / \sigma_{1}\right) \phi_{2}^{1 / 3}},
\end{aligned}
$$

where $\phi_{2}^{1 / 3}=a / \ell, \phi_{2}$ is the volume occupation factor of nanoparticles, and $\tau_{1}=\varepsilon_{0} \varepsilon_{1} / \sigma_{1}$. The negativity of $\varepsilon_{2}$ is necessary to explain the decrease of $\tau$ with the increasing of $\phi_{2}$. The origin of the negativity of $\varepsilon_{2}$ will be discussed in the Section 5 and in the appendix.

The dielectric strength is then given as follows:

$$
\varepsilon(0)-\varepsilon(\infty)=\frac{\left(\varepsilon_{1} \sigma_{2}-\varepsilon_{2} \sigma_{1}\right)^{2} \phi_{2}^{2 / 3}}{\left(\sigma_{1}+\sigma_{2} \phi_{2}^{1 / 3}\right)^{2}\left(\varepsilon_{1}+\varepsilon_{2} \phi_{2}^{1 / 3}\right)},
$$

where the $\varepsilon(\infty)$ is the high frequency limits given as

$$
\varepsilon(\infty)=\varepsilon_{1} .
$$

In (11), $\left(\varepsilon_{1}, \sigma_{1}\right)$ and $\left(\varepsilon_{2}, \sigma_{2}\right)$ look to be mathematically reciprocal each other, but the fact that $\varepsilon(\infty)=\varepsilon_{1}$ and never $\varepsilon(\infty)=\varepsilon_{2}$ demonstrates that they are not reciprocal. 
If we assume that $\sigma_{2} \gg \sigma_{1}$, then we have an approximate equation for the amplification factor or gain, $A_{c}$, such that

$$
\begin{aligned}
A_{c} & =\frac{\{\varepsilon(0)-\varepsilon(\infty)\}}{\varepsilon(\infty)} \\
& =\frac{1}{1+\left(\varepsilon_{2} / \varepsilon_{1}\right) \phi_{2}^{1 / 3}} \\
& =\frac{1}{1-\left|\varepsilon_{2} / \varepsilon_{1}\right| \phi_{2}^{1 / 3}} .
\end{aligned}
$$

We need the negativity of $\varepsilon_{2}$ to have a finite amplification in (13).

The Maxwell-Garnett theory [7, 8] based on the electromagnetic potential theory gives the following equations:

$$
\begin{aligned}
\tau & =\frac{\varepsilon_{0}\left(2 \varepsilon_{1}+\varepsilon_{2}\right)}{2 \sigma_{1}+\sigma_{2}} \\
& =\tau_{1} \frac{1+\varepsilon_{2} / 2 \varepsilon_{1}}{1+\sigma_{2} / 2 \sigma_{1}}, \\
\varepsilon(0)-\varepsilon(\infty) & =\frac{9\left(\varepsilon_{1} \sigma_{2}-\varepsilon_{2} \sigma_{1}\right)^{2} \phi_{2}}{\left(2 \varepsilon_{1}+\varepsilon_{2}\right)\left(2 \sigma_{1}+\sigma_{2}\right)^{2}}, \\
\varepsilon(\infty) & =\varepsilon_{1}\left[1+3 \phi_{2} \frac{\left(\varepsilon_{2}-\varepsilon_{1}\right)}{\left(2 \varepsilon_{1}+\varepsilon_{2}\right)}\right] .
\end{aligned}
$$

A detailed derivation of these equations are given in a paper by Genzel et al. for mainly dealing with surface plasmon effect [12].

It is worth while giving here a note that (14) for the relaxation time has no dependence on $\phi_{2}$ and (15) has a linear dependence on $\phi_{2}$. This behavior does not agree with our experimental results.

The occurrence of the dielectric strength (11) and (15), called the jump caused by the Maxwell-Wagner effect, is originated from the existence of the oscillating extra electric charges appearing in the boundaries between the host medium and the inclusion that is indicated as a point $\mathrm{A}$ in Figure 4(b). A detained analysis of the oscillation of the extra charges and an effective negativity of $\varepsilon_{2}$ will be discussed in the appendix and in the Section 5.

\subsection{The Case Where Alignment Layers Exist: An Interfacial} Effect. We met a necessity to take into account the surface layers in order to get an explanation of the dielectric strength at very low nanoparticle concentration.

Now, we consider the effect of the existence of alignment layers in our dielectric system. We shall write the admittances of the alignment layers as $Y_{3}=G_{3}+i \omega C_{3}$ and that of NLC layer with embedded nanoparticles as $Y=G+i \omega C$, respectively.

Then, the total admittance will be

$$
\begin{aligned}
Y_{T} & =\frac{Y Y_{3} / 2}{Y+Y_{3} / 2} \\
& =\frac{(G+i \omega C)\left(G_{3} / 2+i \omega C_{3} / 2\right)}{\left(G+G_{3} / 2\right)+i \omega\left(C+C_{3} / 2\right)} .
\end{aligned}
$$

The imaginary part of (17) gives the capacitance, and hence the dielectric constant with the dielectric relaxation time reads

$$
\begin{aligned}
\tau & =\frac{\left(C+C_{3} / 2\right)}{\left(G+G_{3} / 2\right)}, \\
\tau & =\frac{C\left(1+C_{3} / 2 C\right)}{G\left(1+G_{3} / 2 G\right)} \\
& =\tau_{1} .
\end{aligned}
$$

Further,

$$
\begin{aligned}
C_{T}(0) & =\frac{1}{2} \frac{G_{3} C+G C_{3}}{G+G_{3} / 2} \\
& \approx \frac{1}{2} C_{3}, \quad \text { if we assume that } \frac{G_{3}}{G} \approx 10^{2}, \\
C_{T}(\infty) & =\frac{1}{2} \frac{C C_{3}}{C+C_{3} / 2} \\
& \approx C .
\end{aligned}
$$

Thus, we have an amplification factor or gain, $A_{c}$, as

$$
\begin{aligned}
A_{c} & =\frac{C_{T}(0)-C_{T}(\infty)}{C_{T}(\infty)} \\
& =\frac{C_{3} / 2-C}{C} .
\end{aligned}
$$

Now, let us evaluate the value of the $A_{c}$. Looking at (20), $A_{c}$ may decrease as the increase of $C$ with the increasing of the nanoparticle concentration. For this reason, the surface effect must be considered only when the concentration of nanoparticles is very low. Then, in this situation, $\tau_{1} \approx C / G$. As appropriate values, we put these values at

$$
\frac{C_{3}}{2 C}=9.2 \times 10^{-1} \approx 1, \quad \frac{G_{3}}{2 G} \approx 1, \quad \text { then } \tau \approx \tau_{1} .
$$

If we take $d=5 \times 10^{-6} \mathrm{~m}$ and $d_{3}=1 \times 10^{-7} \mathrm{~m}$ and $\varepsilon_{3}=$ 2.4 (for a polyimide) and $\varepsilon_{1}=6.5$ (for NLC, $5 \mathrm{CB}$ ), then we put the magnitude of $C$ and $C_{3}$ at $C=\varepsilon_{0} \varepsilon A / d=1.15 \times$ $10^{-5}\left[\mathrm{~F} / \mathrm{m}^{2}\right]$ and $C_{3} / 2=\varepsilon_{0} \varepsilon_{3} A / d_{3}=1.06 \times 10^{-4}\left[\mathrm{~F} / \mathrm{m}^{2}\right]$.

Then, by inserting $C_{3} / 2 C=9.2$ into (20), we get $A_{c}=8.2$.

\section{Comparison between the Experimental Results and Theoretical Calculation}

We have made a theoretical curve fitting to the experimental data of the dielectric relaxation time using (10) by choosing the parameters such that $\tau_{1}=0.3(\mathrm{~s}), \varepsilon_{2} / \varepsilon_{1}=-12.3$, and $\sigma_{2} / \sigma_{1}=5.70 \times 10^{2}$ as shown in Figure 5. The specific dielectric constant of a metal nanoparticle itself is almost unity at zero frequency, and its electrical conductivity is almost zero due to a large electrical depolarization [13]. However, in our case, we measured AC electrical properties of our object and the covering of a core nanoparticle with a diffusion cloud may modify the value of $\varepsilon_{2}$ and $\sigma_{2}$ from those of the bare metal nanoparticle.

The experimental data of the gain, $A_{c}$, takes almost constant value with a slow increase with $\phi_{2}$ as shown in Figure 6. 


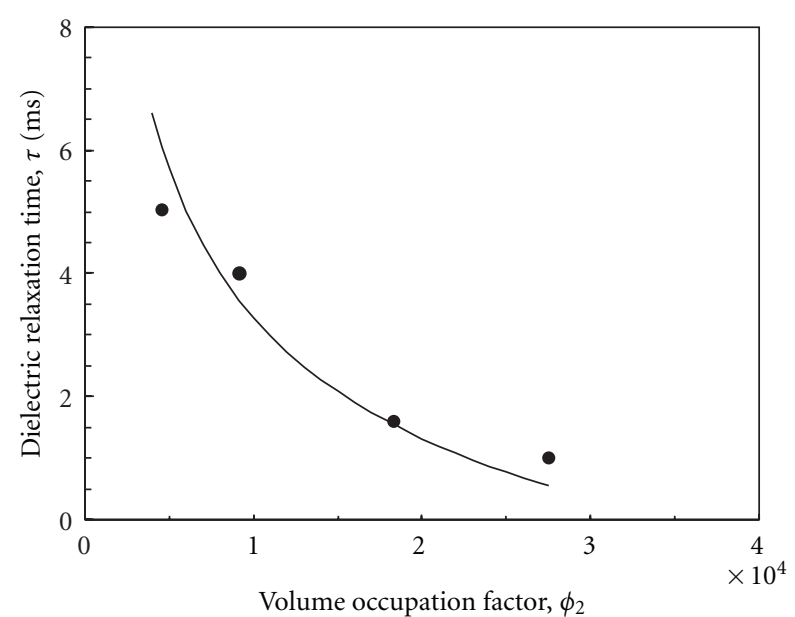

Figure 5: A theoretical curve fitting to the experimental data of dielectric relaxation time in our system using (10).

The value of the gain, $A_{c}$, shown in Figure 6, is not explained in terms of only (13), and further (13) is not compatible with (10) for the relaxation time, $\tau$. This compatibility and the explanation of the gain may be made by considering the following two factors as shown in Figure 6. The gain, $A_{c}$, is whose experimental data are indicated by dots and theoretically calculated values are indicated by a single dotted line (a) in Figure 6. The gain, $A_{c}$, is obtained by superimposing the following two origins: (A) one is the bulk effect containing nanoparticles given by (13) that is indicated by a solid line (b) in Figure 6, and the other (B) is the interfacial effect given by (20) that is indicated by a full dotted line (c) in Figure 6, where the effect (B) gives $A_{c}=8.2$ for the low concentration of nanoparticles, but this effect may fade away as the concentration of nanoparticles increases as is shown by the line (c), since $C$ increases with the increasing of the nanoparticle concentration. Thus, at a high concentration, say, at $0.3 \mathrm{wt} \%$, the effect (A) tends to dominate the effect and produces $A_{c}=12.5$, where we take into account the size of the diffusion cloud is to be slightly larger than that of metal nanoparticles by the factor of $k$. This means that the value of $\phi_{2}^{1 / 3}$, which is given by naked nanoparticles, is replaced by an effective value, that is $k \phi_{2}^{1 / 3}$. If we take $\varepsilon_{2} / \varepsilon_{1}=-12.3$, then the corresponding value of $k$ for the samples from (A) to (D) is commonly $k=1.15$. The meaning of $k$ is that the volume of a bare nanoparticle, $a^{3}$, is replaced by $(k a)^{3}$ due to the covering of each nanoparticle with a diffusion cloud, that is the volume of each nanoparticle covered with the diffusion cloud is $(k a)^{3}$.

As an independent research, we have also conducted a Raman spectroscopy on a Ag nanoparticle-embedded NLC, $5 \mathrm{CB}$ having $\mathrm{C} \equiv \mathrm{N}$ moiety, and the result shows that the NLC molecules in the vicinity of an Ag nanoparticle take a special alignment that is different from the planar alignment in the TN configuration. Thus, the diffusion cloud is considered to be an aligned NLC phase covering metal nanoparticles but has a special molecular alignment and has extra electrical charges, the details of this effect will be published elsewhere [14].

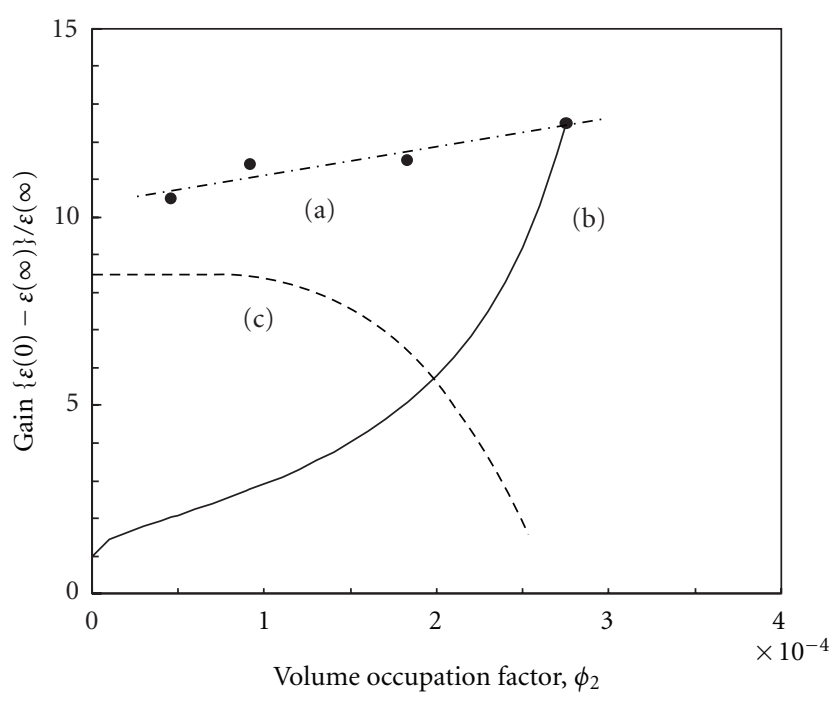

Figure 6: A theoretical curve fitting to the experimental data of the gain in the capacitance that is indicated by a dotted line (a), where this fitting was obtained by superimposition of two origins: one is the bulk effect given by (13) relevant to the nanoparticles and indicated by a solid line (b); the other is the interfacial effect given by (20) and indicated by a full dotted line (c).

It may be considered that the dielectric relaxation time is largely determined by the value of $\left(\sigma_{2} / \sigma_{1}\right) \phi_{2}^{1 / 3}$, where $\sigma_{2} \phi_{2}$ is proportional to the total number of mobile electrons in our system. Formally, the electrical conductivity of a diffusion cloud that contains a nanoparticle must be averaged value of $\sigma_{2}$.

In the appendix, we discuss a detailed explanation of the origin of the apparent negative dielectric constant. By referring to the appendix, let us discuss the possibility and the conditions for producing an effective negative $\varepsilon_{2}$ and also the amplification of the whole capacitance.

In our actual situation, the relevant quantities take the following forms: $C_{1}=\varepsilon_{1} l, G_{1}=\sigma_{1} l, C_{2}=\varepsilon_{2} l$, and $G_{2}=\sigma_{2} l$, and then (A.5) is converted into (22) such that

$$
\frac{\left(\sigma_{2} \phi_{2}^{1 / 3} / \sigma_{1}\right) \tan \Delta \theta}{\omega \tau_{1}}=1+\frac{\varepsilon_{2} \phi_{2}^{1 / 3}}{\varepsilon_{1}} .
$$

Under the condition that $\sigma_{2} / \sigma_{1} \gg 1$.

The left-handed side of (22) must be smaller than unity for giving $\varepsilon_{2}<0$.

As the appropriate values, we put the following quantities at $\phi_{2}=2.57 \times 10^{-4}, \tan \Delta \theta=0.73\left(\Delta \theta=36^{\circ}\right), \sigma_{2} \phi_{2}^{1 / 3} / \sigma_{1}=$ $37, f=70 \mathrm{~Hz}, t_{1}=3 \times 10^{-1}(\mathrm{~s})$, and, by inserting these quantities into (22), then we get the left-handed side of (22) that is to be 0.2 . Thus, from (22), we have $\varepsilon_{2} / \varepsilon_{1}=-12.3$, this agrees very well with that given in the Section 5 . We are also able to claim that the dielectric constant of nanoparticles (inclusions) may behave to have a negative value under the conditions such that

$$
1<\frac{\sigma_{2} \phi_{2}^{1 / 3}}{\sigma_{1}}<\frac{\omega \tau_{1}}{\tan \Delta \theta},
$$

where $\omega \tau<1$ and $\sigma_{2} / \sigma_{1}$ has to exceed a particular value for satisfying the condition given by (23) and $\Delta \theta$ is the delay time 
in the oscillation of the extra charges from the applied AC field.

Basically, the capacitance gain in the actual nematic liquid-crystal (NLC) cells with interfacial polyimide alignment layers comprises a superposition of two origins: one is the type (A) that occurs in the NLC bulk medium embedded with nanoparticles given by (13), and the other is the type (B) that is the M-W effect occurring in the interfacial regions between the alignment layers and the NLC medium given by (20). The surface interfacial effect is dominant when the concentration of the nanoparticle is very low, say $\approx 0.1 \mathrm{wt} \%$. And the effect of the type (B) fades away as increasing the concentration of nanoparticles. At the higher concentration, say, $0.3 \mathrm{wt} \%$, where the dielectric relaxation frequency is high, say, $f_{R}=158.5 \mathrm{~Hz}$, the effect of the type (A) becomes to dominate and produces $A_{c}=12.5$. In this way, the behavior of the gain versus the volume occupation factor of nanoparticles shown in Figure 6 may be qualitatively understood and explained. And further it is shown that the amplification of capacitors may be originated from a special oscillating extra charges appearing on the surface of nanoparticles that is characterized by a finite phase delay in their oscillation to the applied AC electric field and that this oscillation may produce an effective negative dielectric constant of inclusions (nanoparticles).

Even in the traditional theory, the dielectric strength contains $1 /\left(2 \varepsilon_{1}+\varepsilon_{2}\right)$ in the denominator of (15), thus the denominator of this equation must be smaller than unity for giving a finite dielectric strength; then $\varepsilon_{2} \approx-2 \varepsilon_{1}$; thus $\varepsilon_{2} / \varepsilon_{1} \approx-2$. For this reason, the apparent negative dielectric constant is also seen in the traditional theory.

\section{Conclusions}

Through this research, it is experimentally shown that the electrical capacitor containing nematic liquid crystal, $5 \mathrm{CB}$, doped with the metal nanoparticles of $\mathrm{Pd}$, which are particularly covered with diffusion clouds produce an amplification of their capacitance and, hence, that of their dielectric constant. The experimentally obtained value of the amplification factor is about 12.5 at $25^{\circ} \mathrm{C}$ for the concentration of the nanoparticles is $0.3 \mathrm{wt} \%$, where this phenomenon occurs in the $\mathrm{AC}$ frequency region below the dielectric relaxation frequency of $f_{R}=158.7 \mathrm{~Hz}$.

The phenomenon of this amplification has been analyzed by formulating equivalent circuit models not only for the heterogeneous dielectric medium but also for the surface liquid crystal alignment layers. And it is postulated that the dielectric constant, $\varepsilon_{2}$, of the Pd nanoparticle covered with a diffusion cloud has to have a negative value. This may be attributed to an oscillation of the extra electrical charges appearing in the region between each nanoparticle and the surrounding host medium and further that this oscillation is particularly specified by its phase delay to the applied AC field. Bare Pd nanoparticles may not contribute to produce the amplification of the total dielectric constant as shown by Toko et al. [5].

Along with the explanation for the amplification of dielectric constant, we established a compatibility between the amplification of dielectric constant, which is equivalent to the dielectric strength, and the relaxation time. We succeeded in establishing this compatibility in terms of the above-mentioned equivalent circuit formulae and by taking the effective size of each nanoparticle to be $(k a)^{3}$, where $a^{3}$ is the size of each bare metal nanoparticle and $k$ is to be $k=1.15$.

\section{Appendix}

\section{About the Apparent Negative Dielectric Constant of Nanoparticles}

The amplification occurring in a capacitor filled with a nematic liquid crystal medium doped with the nanoparticles of metal, which are covered with an ionic diffusion cloud, may be caused by an effective negative dielectric constant of all the metal nanoparticles covered with the ionic diffusion cloud. This appendix shows the origin of the amplification in the capacitance that may be basically attributed to the behavior of oscillating extra charges, which appear in the region of the boundaries between the two media (the host media and inclusion) for an applied AC field. Each of medium is characterized by $C_{1}, G_{1}$ and $C_{2}, G_{2}$, where $C_{1}$ and $G_{1}$ are the capacitance and conductance of the host medium and $C_{2}$ and $G_{2}$ are those of the inclusion (nanoparticles), respectively.

For an applied voltage $V=V_{0} \sin (\omega t)$, we have extra oscillating charges, $\Delta q$, appearing at the boundary between the host medium and an inclusion and with a finite a phase delay $\Delta \theta$ to the applied voltage that is expressed such that

$$
\begin{aligned}
\Delta q & =q_{2}-q_{1} \\
& =\frac{G_{1} G_{2} \Delta \tau}{\left(G_{1}+G_{2}\right)\left\{1+(\omega \tau)^{2}\right\}^{1 / 2}} \cdot\left\{V_{0} \sin (\omega t-\Delta \theta)\right\},
\end{aligned}
$$

in the steady state, where $q_{2}$ and $q_{1}$ are the charge in each medium, and $\tau_{1}$ and $\tau$ are given as

$$
\Delta \tau=\frac{C_{1}}{G_{1}}-\frac{C_{2}}{G_{2}}, \quad \tau=\frac{C_{1}+C_{2}}{G_{1}+G_{2}} .
$$

Furthermore, we have

$$
\tan \Delta \theta=\omega \tau,
$$

where $\Delta \theta=\pi / 4$ gives $\omega \tau=1$; thus, $\Delta \theta$ must be $0<\Delta \theta<$ $\pi / 4$.

Now, let us discuss the condition for producing an effective negative value of $C_{2}$ and also an amplification of total dielectric strength using (A.3), which will be rewritten by taking into account that $\tau=\left(C_{1}+C_{2}\right) /\left(G_{1}+G_{2}\right)$ such that

$$
\tau=\frac{\tan \Delta \theta}{\omega}=\frac{C_{1}+C_{2}}{G_{1}+G_{2}}=\frac{C_{1}}{G_{1}} \cdot \frac{\left(1+C_{2} / C_{1}\right)}{\left(1+G_{2} / G_{1}\right)} .
$$

Then, we have (A.5) as follows:

$$
\frac{\left(G_{2} / G_{1}\right) \tan \Delta \theta}{\omega \tau_{1}}=1+\frac{C_{2}}{C_{1}},
$$

where $\tau_{1}=C_{1} / G_{1}$, and we assume that $G_{2} / G_{1} \gg 1$. 
The condition for getting an effective negative $C_{2}$ is such that

$$
\frac{\left(G_{2} / G_{1}\right) \tan \Delta \theta}{\omega \tau_{1}}<1 .
$$

For satisfying this condition, $G_{2} / G_{1}$ has to satisfy the condition

$$
1<\frac{G_{2}}{G_{1}}<\frac{\omega \tau_{1}}{\tan \Delta \theta} .
$$

If we substitute appropriate values for the relevant quantities into (A.7) in such a way that $\tan \Delta \theta=0.7, \tau_{1}=3 \times 10^{-1}(\mathrm{~s})$, $f=50 \mathrm{~Hz}$, then, we have

$$
1<\frac{G_{2}}{G_{1}}<134 .
$$

If we insert $G_{2} / G_{1}=20$ into (A.5), then we have

$$
\frac{\left(G_{2} / G_{1}\right) \tan \Delta \theta}{\omega \tau_{1}}=0.15 \text {. }
$$

From (A.5) and (A.9), we have

$$
\frac{C_{2}}{C_{1}}=-0.85 \text {. }
$$

Thus, $C_{2}=-0.85 \times C_{1}$.

In this way, the negativity of $C_{2}$ is obtained. 1.

Now, we shall discuss the amplification, $A_{c}$, for $G_{2} / G_{1}>$

$A_{c}$ is defined and given as follows:

$$
\begin{aligned}
A_{c} & =\frac{C(0)-C(\infty)}{C(\infty)} \\
& =\frac{C_{1}}{C_{1}+C_{2}} \\
& =\frac{1}{1-\left|C_{2}\right| / C_{1}},
\end{aligned}
$$

for $C_{2} / C_{1}=-0.85$, we have

$$
A_{c}=\frac{1}{1-0.85}=\frac{1}{0.15} .
$$

Thus, we get $A_{c}>1$ such that

$$
A_{c}=6.7
$$

Notice. For the expression of the amplification, a similar equation such as (A.11) commonly appears in the equation of almost all kinds of electronic amplifiers in such a way that $1 /(1-\alpha)$, where $\alpha$ is close to unity (e.g., a formulae in a Book by Sher [15]).

\section{Acknowledgments}

The authors are indebted for the Grant METI Regional Revitalization Consortium R\&D Project, H16, and 17 S6001 and that of MEXT City Area Collaboration "Nano LCs" H18$\mathrm{H} 20$.

\section{References}

[1] B. Corain, G. Schmid, and N. Toshima, Eds., Metal Nanoclusters in Catalysis and Material Science: The Issue of Size-Control, Elsevier, Amsterdam, The Netherlands, 2008.

[2] Y. Shiraishi, N. Toshima, K. Maeda, H. Yoshikawa, J. Xu, and S. Kobayashi, "Frequency modulation response of a liquidcrystal electro-optic device doped with nanoparticles," Applied Physics Letters, vol. 81, no. 15, pp. 2845-2847, 2002.

[3] H. Yoshikawa, K. Maeda, Y. Shiraishi et al., "Frequency modulation response of a tunable birefringent mode nematic liquid crystal electrooptic device fabricated by doping nanoparticles of Pd covered with liquid-crystal molecules," Japanese Journal of Applied Physics Part 2, vol. 41, no. 11 B, pp. L1315-L1317, 2002.

[4] T. Miyama, J. Thisayukta, H. Shiraki et al., "Fast switching of frequency modulation twisted nematic liquid crystal display fabricated by doping nanoparticles and its mechanism," Japanese Journal of Applied Physics Part 1, vol. 43, no. 5 A, pp. 2580-2584, 2004.

[5] Y. Toko, T. Takahashi, K. Miyamoto et al., "Fast electro-optical response at low temperature for metal nanoparticle embedded STN-LCDs (invited paper)," Journal of the Society for Information Display, vol. 16, no. 9, pp. 957-960, 2008.

[6] S. Kobayashi, T. Miyama, N. Nishida et al., "Dielectric spectroscopy of metal nanoparticle doped liquid crystal displays exhibiting frequency modulation response," IEEE/OSA Journal of Display Technology, vol. 2, no. 2, pp. 121-128, 2006.

[7] J. C. Maxwell-Garnett, "Colours in metal glasses and in metallic films," Philosophical Transactions, vol. 203, pp. 385-420, 1904.

[8] J. C. Maxwell-Garnett, "Colours in metal glasses, in metallic films, and in metallic solutions. II," Philosophical Transactions, vol. 205, pp. 237-288, 1906.

[9] J. C. Maxwell, Treatise on Elekctricity and Magnetism, vol. 1, Dover, New York, NY, USA, 1945.

[10] K. W. Wagner, Erklarung Arch: Elektrotech, vol. 2, p. 371, 1914.

[11] S. Oka, Theory of Solid Dielectrics, Maruzen, 1956.

[12] L. Genzel, T. P. Martin, and U. Kreibig, "Dielectric function and plasma resonances of small metal particles," Zeitschrift für Physik B, vol. 21, no. 4, pp. 339-346, 1975.

[13] C. Kittel, Introduction to Solid State Physics, John Wiley \& Sons, 7 th edition, 1996.

[14] S. Hashimoto et al., "Enhanced raman spectra on 5CB covered Ag nanoparticles," to be published.

[15] M. Sher, Physics of Semiconductor Devices, chapter 3, Prentice Hall, 1990. 

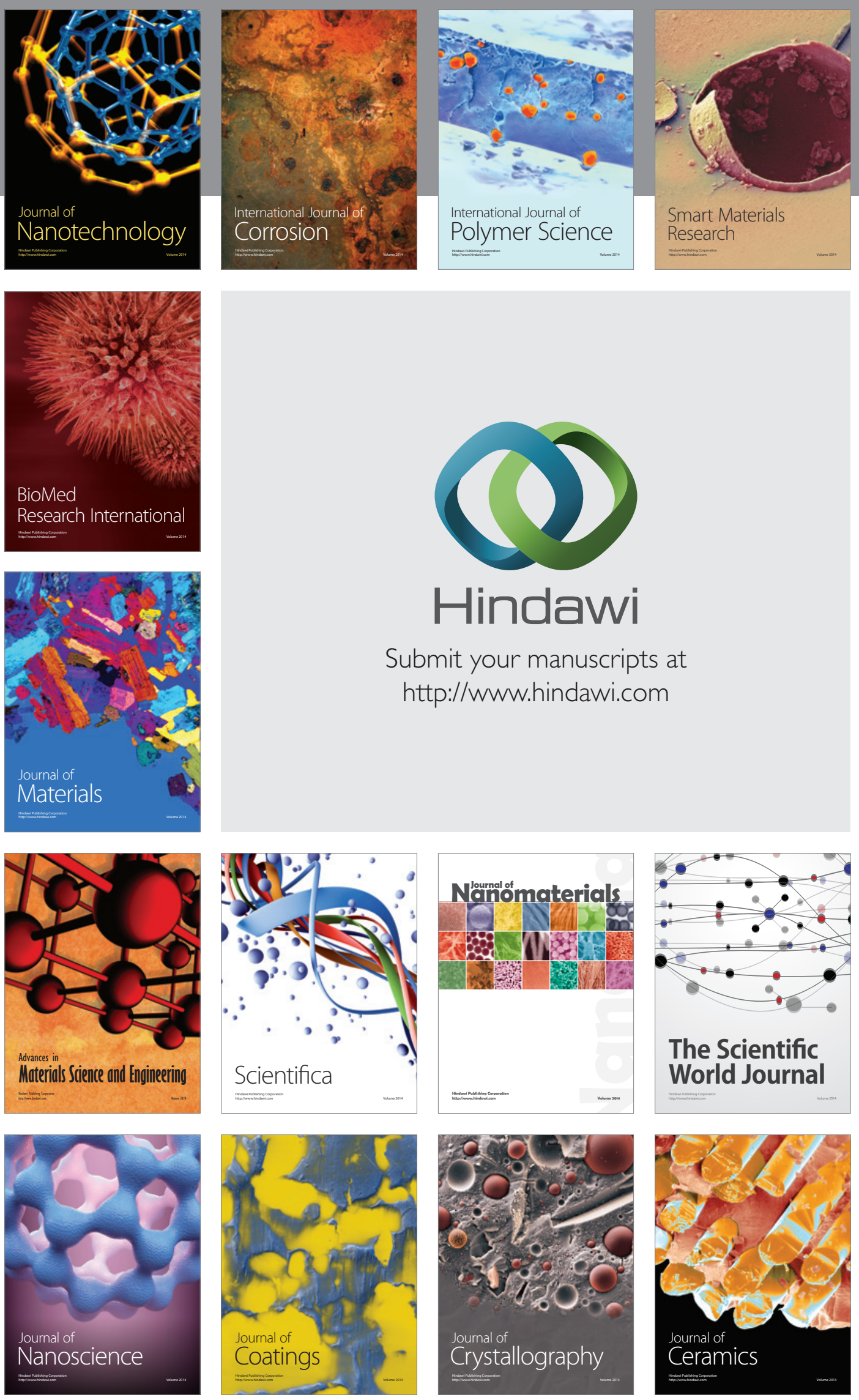

The Scientific World Journal

Submit your manuscripts at

http://www.hindawi.com

\section{World Journal}

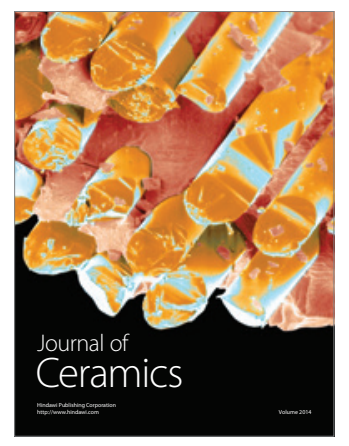

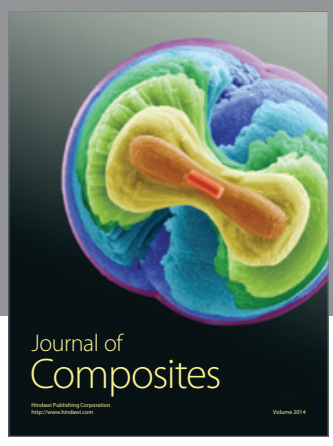
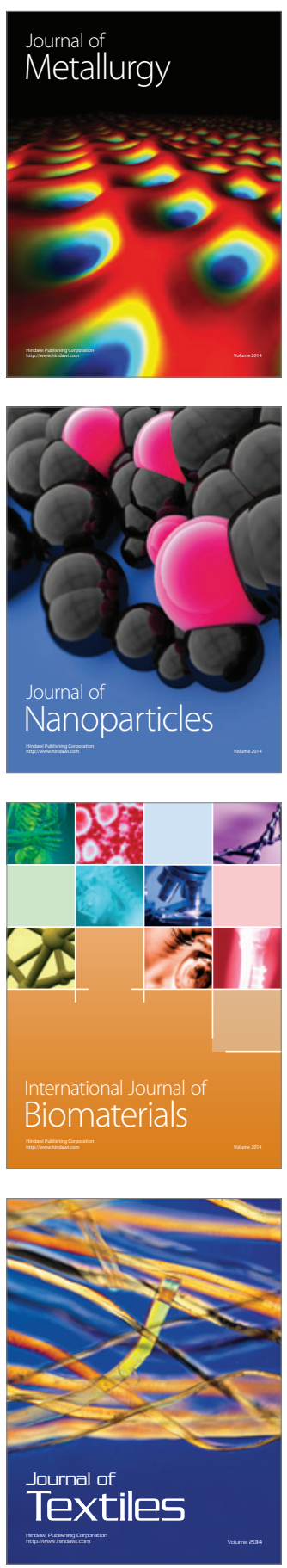